\title{
Combination of Genetics and Nanotechnology for Down Syndrome Modification: A Potential Hypothesis and Review of the Literature
}

\author{
Alireza TAFAZOLI ${ }^{1,2}$, Farkhondeh BEHJATI ${ }^{3}$, Dariush D. FARHUD ${ }^{4,5}$, *Mohammad \\ Reza ABBASZADEGAN ${ }^{6}$
}

1. Department of Analysis and Bioanalysis of Medicine, Faculty of Pharmacy with the Division of Laboratory Medicine, Medical
University of Bialystok, Bialystok, Poland

2. Department of Endocrinology, Diabetology and Internal Medicine, Clinical Research Center, Medical University of Bialystok, Bialystok, Poland

3. Genetics Research Center, University of Social Welfare and Rehabilitation Sciences, Tehran, Iran

4. School of Public Health, Tehran University of Medical Sciences, Tehran, Iran

5. Department of Basic Sciences, Iranian Academy of Medical Sciences, Tehran, Iran

6. Medical Genetics Research Center, Mashhad University of Medical Sciences, Mashhad, Iran

*Corresponding Author: Email: Abbaszadeganmr@mums.ac.ir

(Received 16 Feb 2018; accepted 07 Jun 2018)

\begin{abstract}
Down syndrome (DS) is one of the most prevalent genetic disorders in humans. The use of new approaches in genetic engineering and nanotechnology methods in combination with natural cellular phenomenon can modify the disease in affected people. We consider two CRISPR/Cas9 systems to cut a specific region from short arm of the chromosome 21 (Chr21) and replace it with a novel designed DNA construct, containing the essential genes in chromatin remodeling for inactivating of an extra $\mathrm{Chr} 21$. This requires mimicking of the natural cellular pattern for inactivation of the extra X chromosome in females. By means of controlled dosage of an appropriate Nano-carrier (a surface engineered Poly D, L-lactide-co-glycolide (PLGA) for integrating the relevant construct in Trisomy 21 brain cell culture media and then in DS mouse model, we would be able to evaluate the modification and the reduction of the active extra $\mathrm{Chr} 21$ and in turn reduce substantial adverse effects of the disease, like intellectual disabilities. The hypothesis and study seek new insights in Down syndrome modification.
\end{abstract}

Keywords: Down syndrome; CRISPR/Cas9; Designed DNA construct; Poly D L-lactide-co-glycolide (PLGA); Nano-carrier; Chromosome 21 inactivation

\section{Introduction}

Down syndrome (DS) is one of the most famous and prevalent genetic diseases in medical history $(1,2)$. The frequency of DS is one in every 660 individuals in different populations (3). Although there are some verified prenatal tests, which indicate the presence of the disease with great accuracy and specificity (4-6), it is still one of the most worldwide consideration to control by health care providers and the affected people themselves, especially in the Middle East and third world countries. Moreover many previous studies have tried to introduce prevention/treatment issues. To date, many treatments or modifying procedures including general and symptomic interventions have been suggested by investigators (7-11). Here we proposed a method, which can potentially reduce the main effects and clinical features in DS significantly. 
Referring to published papers, we can find numerous studies about more important genes that show higher impact in creating DS clinical manifestations. Investigations on Down patients with partial trisomy and/or mosaicism form, revealed a $1.6 \mathrm{Mb}$ critical region on chromosome $21 \mathrm{q} 22$ with highest amount of transcription in DS (12, 13). This area is called DS Critical Region (DSCR). Most of the genes, located in DSCR are related to development of the brain and play an essential role in learning and cognition processes and loss of them lead to neuropathologic issues in DS. Even some new investigations restricted the DSCR region to a very small area, duplicated in all DS patients (14). Hence, consideration of lower number of genes, which are more specific for DS phenotypes, seems to be more logical for modifications to correct adverse phenotypes.

\section{Principles of $\boldsymbol{X}$ chromosome inactivation}

In order to progress on this hypothesis, we need to indicate more detailed facts about the cellular regulators in women $\mathrm{X}$ chromosome inactivation process. Generally, there is a natural cellular intelligence for inactivating all the defined extra $\mathrm{X}$ chromosome in human cells on the different condition, including a normal woman's cell or any numerical chromosome disorders like XXX women. Inactivation begins in fetal age by the expression of a non-coding RNA (Xist) on the long arm of target X chromosome. Accumulation and spreading of Xist from X inactivation center (XIC) make transcription silencing take place in other parts of $\mathrm{X}$ chromosome. The facts indicate the essential role of chromatin modifications and epigenetic changes in gene silencing on $\mathrm{Xi}(15,16)$. Even the best way for the modifications of gene expression and activation could be the design and creation of new epigenetic changes, which can act as an on/off switch for it, not making the alteration in gene sequence itself (17). Therefore the identification and administration of cell products participated in chromatin modifications, epigenetic marks in different cells, and performing the inactivation procedure, would be essential for understanding the inactivation mechanisms and possible use of them as a potential modification tool.
Short overview on CRISPR/Cas9 system Using CRISPR/Cas9 system to cut the human genome was developed in early 2013 (18-20). Then lots of publications appeared in this field, introducing various functions for different types of the system. The main utilities could be summarized as 1 . Targeting a specific sequence and cutting relevant site, 2. Targeting, cutting the position and replacing it with desired sequence, 3 . Attachment of the bro$\mathrm{ken} /$ mutated Cas9 enzyme to the favorite sequence and blocking the transcription or silencing the relevant gene by limiting the RNA polymerase activity, 4. Attachment of the inactive enzyme to an activator protein with a duty on stimulating gene expression results in controlled gene switch on/off (using different RNA guide for different cell lines), 5. Rewriting and making new epigenetic alterations in specific sites by broken/mutated Cas9, 6. Identification of gene function and regulatory regions in genome, and 7. Produce new animal models for various diseases by creating mutations using the CRISPR/Cas9 tool (21-24). CRISPR system aims its target by a single-stranded guide complementary RNA (sgRNA), replaced with synthetic exogenous RNA, and cut the relevant sequence by Cas 9 enzyme. The generated double-stranded break (DSB) position can be treated using two alternative ways: a) the system deletes or inserts nucleotides randomly, using Non-Homologous End Joining (NHEJ) repair mechanism, which can lead to target gene sequences disruption. b) insert an external DNA as a template, with homology sequences between both $5^{\prime}$ and $3^{\prime}$ sides of the template and wild type target sequence, for mutation correction in DSB site. The latter is called Homology-Directed Repair (HDR), which can result in the alteration of target gene sequence and function $(19,25)$. In this way, researchers can perform an extensive range of sequence changing from a single nucleotide to a large insertion or replacement, which would be a permanent alteration in the genome too (26). The gRNA is always designed in a way to introduce DSB to the desired sequence (21). Moreover, just one suitable gRNA would be enough for making a DSB in target sequence and subsequent HDR, without any consideration for insert size (27). Even by using a 
number of gRNA simultaneously, DSB in large fragments of the genome could be achieved.

\section{In-vivo delivery of CRISPR/Cas9 for thera- peutic purposes}

The next important step is introducing a practical way for in vivo delivery purposes. Different types of CRISPR/CAs9 system introduced for transferring into the target cells, comprising Cas9 gene + gRNA gene (gene-based transferring method), Cas9 mRNA + synthetic gRNA (RNA based delivery method), and Cas9 protein + synthetic gRNA. The latter produces a RNP complex and is considered as a protein-based delivery method, which shows many advantages upon two other forms. This type of delivery system demonstrates a lot of benefits i.e. very low off-target ratio, absence of any immunogenicity, no risk for insertional muatgenesis (as it could happen with Cas9 gene transferring to the cell), and more efficiency in editing task (28). However, direct transporting of the Cas9 protein plus other components including gRNA and template DNA into the target cells should utilize a confident and specific approach. Today, nano carriers are proved to be the best way for delivering the CRISPR/Cas9 components in cells. Nanoparticle (NP) vector, coated with cationic polymer (polyethylenimine), has been used for transferring the Cas9 protein and gRNA in cancer model mouse before (29). Moreover, engineered lipid base NPs optimized for specific delivery, applied to convey CRISPR components with high-efficiency percentage (30).

\section{The Hypothesis and study design}

Here we explore the hypothesis of applying two distinct CRISPR/Cas9 systems simultaneously in a protein-based delivery method to both brain cell culture and DS mouse model. Selecting the brain cells is unavoidable since investigations indicate that most of the genes in DSCR area show a role in learning and cognition processes. Additionally, a mouse model of the disease is the second choice for examination of our theory. The expression and silencing of genes in the inactivated X chromosome of the mouse is controlled by single genes (31). This makes the mouse a suitable model for our inquiry since we consider some specific genes for silencing of other single genes (Table 1). In our opinion, for modification of the DS and attenuation of the main intellectual disability effects, we can inactivate the extra Chr21 in some specific tissues in patients. For this purpose, we assume using two different manipulating CRISPR/Cas9 tools including 1. A Cas9 protein + two different sgRNAs for cutting off two ends of DSCR region in Chr21 (gene deletion approach) and 2. A Cas9 protein + a sgRNA + a template dsDNA to cut a nonfunctional region of the centromere-proximal half of 21q (32), and replace it with a newly designed DNA construct, which contains some major genes in chromatin remodeling and epigenetic effects for the inactivation of one extra Chr21 (Fig. 1).

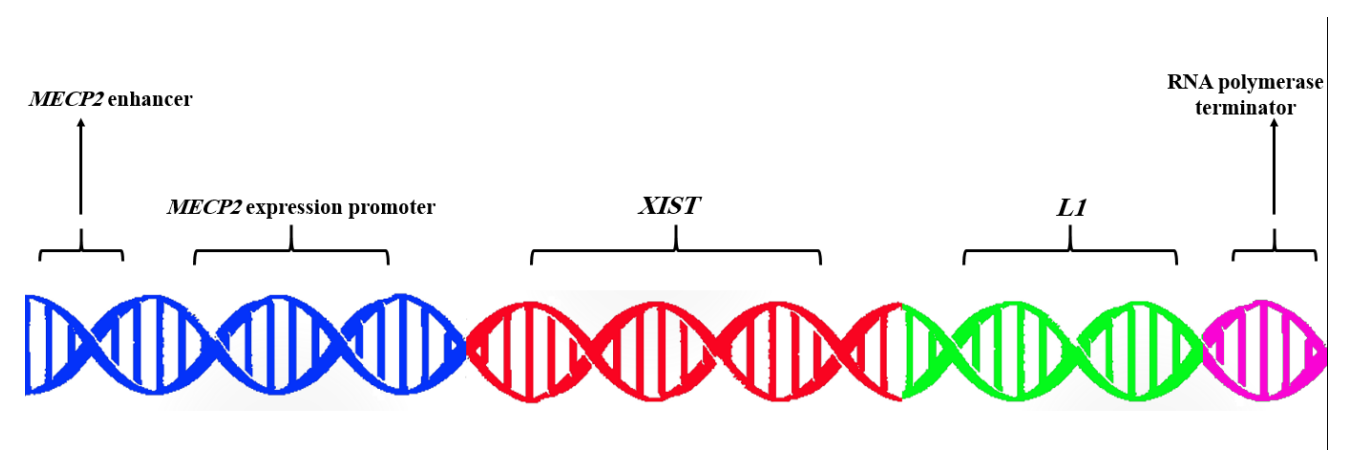

Fig. 1: The designed DNA construct for replacing specific, nonfunctional position on Chr21. This includes an enhancer for brain cells special promoter (i.e. MECP2 promoter), MECP2 expression promoter, XIST gene as a main epigenetic modifier factor for inducing inactivation on relevant chromosome, and a long non-coding RNA gene like L1 gene on behalf of a particular element for promoting Xist RNA function. Also, an RNA polymerase terminator gene would be necessary in order to control the transcription activities 
The latter represents a gene insertion or gene knock-in system by HDR method for inactivating procedure of Chr21. In this way, first, the DSCR region would be omitted and then by insertion of the regulatory DNA construct, whole Chr21 would be exposed to inactivation. In fact, repressing of extra Chr21 and its components would be guaranteed by two subsequent hits.
"Make assurance double sure"! We expect inactivation process to occur in other chromosomes too because females' $\mathrm{X}$ chromosome is not the only inactivated chromosome example in human body. Investigations demonstrated that the inactivation also could be seen in males' germ cells as meiotic sex chromosome inactivation (MSCI) form (33).

Table 1: List of expressed and silenced Genes on extra chromosome 21 in Down Syndrome patients

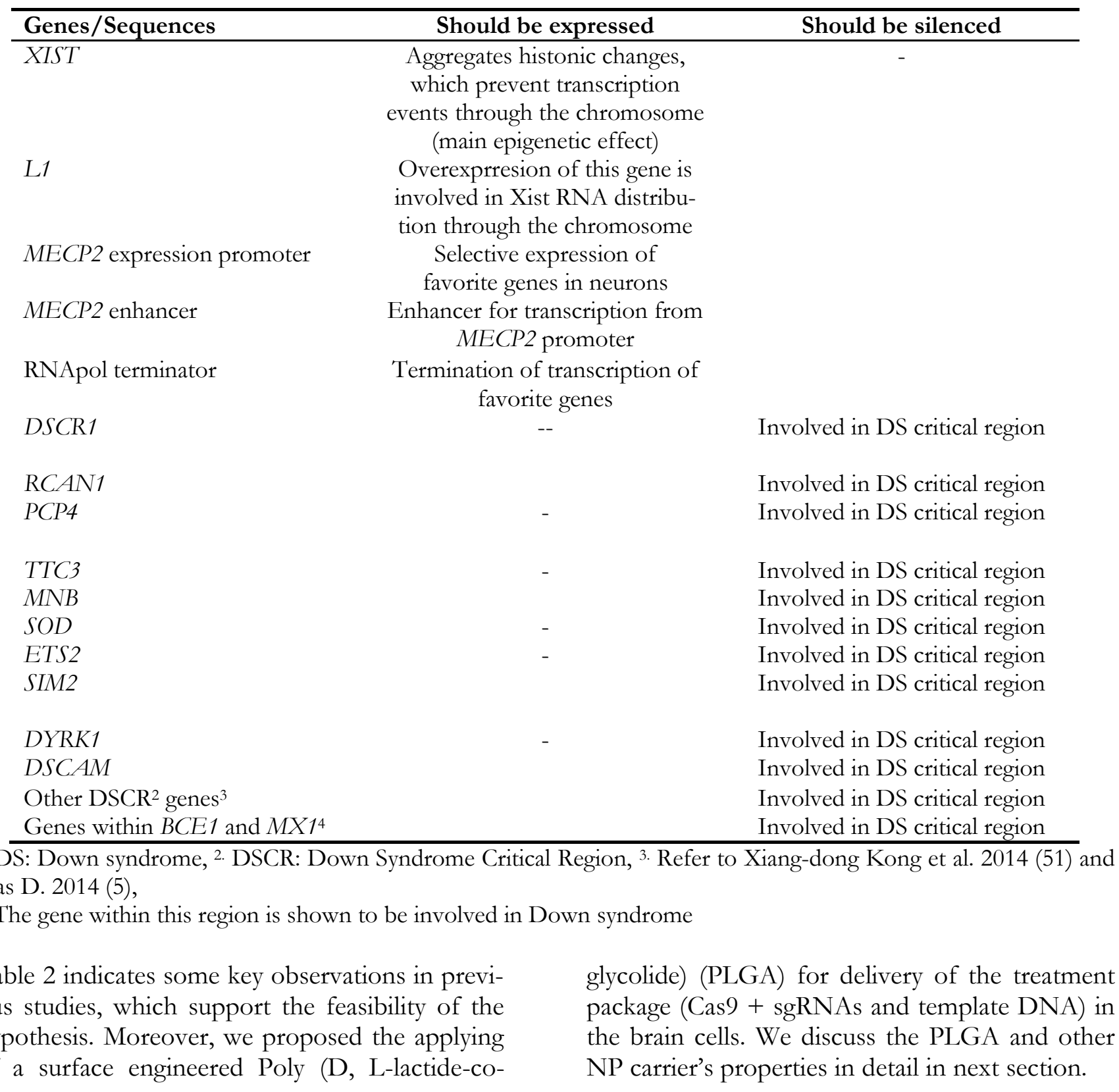


Table 2: List of studies, which provide evidences for supporting the hypothesis

\begin{tabular}{|c|c|}
\hline Reference & Research results \\
\hline Song R. et al. 2009 & Inactivation can be seen in other chromosomes too \\
\hline Li et al. 2015 & Knock-in of full exon 44 in DMD gene by CRISPR methodswith $75 \%$ efficiency \\
\hline Ousterout et al. 2015 & $\begin{array}{l}\text { All the region between exons } 45 \text { to } 55 \text { in } D M D \text { gene have been cut with CRISPR suc- } \\
\text { cessfully (this was a relatively large amount of } D N A \text {, omitted from the genome) }\end{array}$ \\
\hline Yin H. et al. 2014 & $\begin{array}{l}\text { Efficiency of } 1 \text { in } 250 \text { cells is detected by using non-viral delivery methods for CRISPR } \\
\text { system }\end{array}$ \\
\hline Mout R. et al. 2017 & High efficiency for delivery of CRISPR/Cas9 components with Nano-carriers reported \\
\hline $\begin{array}{l}\text { Slaymaker I.M. et al. } 2016 \text { and } \\
\text { Kleinstiver B.P. et al. } 2016\end{array}$ & $\begin{array}{l}\text { Engineered Cas9 with high specificity for target cells and reduced off-target effects de- } \\
\text { scribed }\end{array}$ \\
\hline Yang Su et al. 2017 & CRISPR/Cas9 editing alleviates Huntingon's symptoms in model mice \\
\hline
\end{tabular}

\section{Results and Discussion}

The designed DNA construct in this hypothesis, induce the inactivation procedures for extra Chr21 in the patients. The inactivation will follow and portray the events, which occur for extra X chromosomes in females. This, activate a process, which rewrites the epigenetic marks for specific tissue. Here we utilize a XIST gene in our construct because it will stimulate the aggregation of histonic changes for expanding prevention of transcription events in whole relevant chromosome $(34,35)$. Moreover, using a particular long non-coding RNA (lncRNA) like Line-1 (L1) would be beneficial and strengthens Xist function, as previous studies depict the expression and even overexpression of some lncRNAs which modifies the distribution of Xist RNA in relevant chromosome $(16,36)$. Chromatin analysis methods are available for detection of genes, escaped from inactivation system and could be done after our induced inactivation accomplished (15). Moreover, new approaches like chromosome specific cDNA-array have been used for this purpose in mouse brain cells in recent years $(37,38)$. Here, we need some comprehensive and accurate techniques for evaluation of inactivation in targeted additional Chr21 in the experiment. Nowadays, different methods including differential expression analysis according to cDNA microarray expression data, Spearman correlation coefficient for analysis of modification in genes and pathways (https://sysbiowiki.soe.ucsc.edu/)
(39), and wide gene expression analysis for genes on Chr21 in DS patients (40, 41).

Next, we should employ a brain cell culture media and a DS mouse model for examination of the designed construct. There are some standard brain cells media that are routinely used in experiments $(42,43)$. However, more advanced results could be achieved if brain cells, obtained from brain biopsy of DS patients, are directly used in cell culture media (21). For finding the suitable and appropriate mouse model of DS, we can refer to J. Braudeau et al. and Miyamoto K. publications $(44,45)$. After that, sending the modification materials to the brain cells must be accomplished. Generally, intraperitoneal injection is an effective approach for brain cells targeting in mouse model (46).

Subsequent effort will be allocated for entering the appropriate concentrations of Cas9 protein and its properties to the target cells. Recently, engineered Cas9 containing an oligo glutamic acid tag (E-tag), with negative charge has been packaged with sgRNA and are entered in a Gold nanoparticle (AgNP) for direct transfer into the cytoplasm and nucleus space of host cells. The in vitro efficacy was reported for $90 \%$ of cells (47). Moreover, engineered Cas9 with high specificity for target cells and reducing off-target effects has been used before $(48,49)$. Protein-based CRISPR delivery displays low off-target rate in experiments. Finally, by the administration of an appropriate dosage of doxycycline component, Cas9 activation can be controlled in cells (50). 
These and many other efforts in this field, show the extreme progress in CRISPR and Nano carrier-mediated methods for human body cells manipulation (Table 2). Here, we recommend using two distinct CRISPR components for complete inactivation achievement. Theoretically, it would be easier and less difficult in laboratory to just aim, cut, and omit the overexpressed genes in DS cells instead of employing an extra Cas9 system with template DNA, which utilizes HDR process for replacing new genes. However, investigations have shown creating some DSB in DNAs without replacing any material, increasing the chance of unwanted chromosomal rearrangements (21). Hence, advanced manipulation methods just like this hypothesis and using new discoveries in cell reprogramming control like adding ZSCAN4 gene in the construct, would be essential to guarantee the efficacy rate of the system.

Moreover, in this hypothesis, we must get the interfering tools to specific cells in the brain. Using special NPs like Solid lipid Nano particles (SLNs), PLGA, and etc. are the suitable Nano carriers for brain cells delivery $(51,52)$. Modified and engineered surfaces of these and other NPs can perform the targeting process more specifically. For example, altered surface of Poly (nbutyl cyanoacrylate) with polysorbate 80 , demonstrated its ability to pass through blood-brain barrier invivo (53). Recent studies also use the PLGA, which when engineered with natural cellmembrane derived lipid vesicles (nanoghosts), are more successful target specificity for the carrier (54). These investigations on delivery rate of Nano assemblies show that engineering NPs with particular peptides or lipids on their surfaces can guarantee the specific delivery to the target tissues, both in vitro and in vivo.

\section{Conclusion}

This hypothesis proposes a knowledge-based approach to attenuate DS main effects in suffered patients. Besides, the introduced technique has the ability to focus on some specific genes like those involved in cardiac and septal defects and even cancer-prone genes in DS patients (55). Future steps can be modifying of the particular alleles for DS genes in different populations and ethnic groups. This approach brings new hope for DS patients, especially those, who indicate no brain dementia or show dementia in their youth or middle age and promises a better life for them. In addition, main future efforts would be focused on the entering the appropriate dosage of the materials, especially the Cas9 protein, and using the comprehensive assessment methods and functional evaluations of the entire genome after the treatment, by means of wide-ranging techniques like whole genome sequencing (WGS) and other assays.

\section{Ethical considerations}

Ethical issues (Including plagiarism, informed consent, misconduct, data fabrication and/or falsification, double publication and/or submission, redundancy, etc.) have been completely observed by the authors.

\section{Conflict of interest}

The authors declare that there is no conflict of interest.

\section{References}

1. Blank M, Fuerst PG, Stevens B et al (2011). The Down syndrome critical region regulates retinogeniculate refinement. J Neurosci, 31:5764 5776.

2. Chatterjee A, Dutta S, Sinha S, Mukhopadhyay K (2011). Exploratory investigation on functional significance of ETS2 and SIM2 genes in Down syndrome. Dis Markers, 31:247-257.

3. Jones KL, Jones MC, Del Campo M (2013). Smith's Recognizable Patterns of Human Malformation EBook. ed. Elsevier Health Sciences.

4. Wald NJ, Huttly WJ, Hackshaw AK (2003). Antenatal screening for Down's syndrome with the quadruple test. Lancet, 361:835-836.

5. Palomaki GE, Deciu C, Kloza EM et al (2012). DNA sequencing of maternal plasma reliably identifies trisomy 18 and trisomy 13 as well as Down syndrome: an international collaborative study. Genet Med, 14:296-305.

6. Palomaki GE, Kloza EM, Lambert-Messerlian GM et al (2011). DNA sequencing of maternal 
plasma to detect Down syndrome: an international clinical validation study. Genet Med, 13:913-920.

7. Ali FE, Al-Busairi WA, Al-Mulla FA (1999). Treatment of hyperthyroidism in Down syndrome: case report and review of the literature. Res Dev Disabil, 20:297-303.

8. Lee J-E, Jang H, Cho E-J, Youn H-D (2009). Down syndrome critical region 1 enhances the proteolytic cleavage of calcineurin. ExpMolMed, 41:471-477.

9. Rissman RA, Mobley WC (2011). Implications for treatment: GABAA receptors in aging, Down syndrome and Alzheimer's disease. J Neurochem, 117:613-622.

10. Das D, Phillips C, Hsieh W et al (2014). Neurotransmitter-based strategies for the treatment of cognitive dysfunction in Down syndrome. Prog Neuropsychopharmacol Biol Psychiatry, 54:140-148.

11. McCabe LL, McCabe ER (2013). Down syndrome and personalized medicine: Changing paradigms from genotype to phenotype to treatment. Congenit Anom (Kyoto), 53:1-2.

12. Shapiro B (1999). The Down syndrome critical region. In: The Molecular Biology of Down Syndrome. Ed(s): Springer, pp. 41-60.

13. Hamosh Ada SPM (2010). Down syndrome critical region gene 4 https://www.omim.org/entry/604829

14. Pelleri MC, Cicchini E, Locatelli $C$ et al (2016). Systematic reanalysis of partial trisomy 21 cases with or without Down syndrome suggests a small region on 21q22. 13 as critical to the phenotype. Hum Mol Genet, 25:2525-2538.

15. Berletch JB, Yang F, Disteche CM (2010). Escape from $\mathrm{X}$ inactivation in mice and humans. Genome Biol, 11:213.

16. Berletch JB, Yang F, Xu J, Carrel L, Disteche CM (2011). Genes that escape from X inactivation. Hum Genet, 130:237-245.

17. Rots M (2014). CRISPR-Cas, Zinc Fingers and Epigenetic Editing with Dr. Marianne Rots. Epigenetics, 2.

18. Cong L, Ran FA, Cox D, Lin S et al (2013). Multiplex genome engineering using CRISPR/Cas systems. Science, 339:819-823.

19. Mali P, Yang L, Esvelt KM et al (2013). RNAguided human genome engineering via Cas9. Science, 339:823-826.
20. Jinek M, East A, Cheng A et al (2013). RNAprogrammed genome editing in human cells. Elife, 2:e00471.

21. Men K, Duan X, He Z et al (2017). CRISPR/Cas9mediated correction of human genetic disease. Sci China Life Sci. 60:447-457.

22. Qi LS, Larson MH, Gilbert LA et al (2013). Repurposing CRISPR as an RNA-guided platform for sequence-specific control of gene expression. Cell, 152:1173-1183.

23. Zalatan JG, Lee ME, Almeida R et al (2015). Engineering complex synthetic transcriptional programs with CRISPR RNA scaffolds. Cell, 160:339-350.

24. Kiani S, Beal J, Ebrahimkhani MR et al (2014). CRISPR transcriptional repression devices and layered circuits in mammalian cells. Nat Methods, 11:723-726.

25. Song B, Fan Y, He W et al (2015). Improved hematopoietic differentiation efficiency of genecorrected beta-thalassemia induced pluripotent stem cells by CRISPR/Cas9 system. Stem Cells Dev, 24:1053-1065.

26. Savić N, Schwank G (2016). Advances in therapeutic CRISPR/Cas9 genome editing. TranslRes, 168:15-21.

27. Yoshimi K, Kaneko T, Voigt B, Mashimo T (2014). Allele-specific genome editing and correction of disease-associated phenotypes in rats using the CRISPR-Cas platform. Nat Commun, 5:4240.

28. Komor AC, Badran AH, Liu DR (2017). CRISPRbased technologies for the manipulation of eukaryotic genomes. Cell, 169(3):559.

29. Sun W, Ji W, Hall JM et al (2015). Self-Assembled DNA Nanoclews for the Efficient Delivery of CRISPR-Cas9 for Genome Editing. Angen Chem Int Ed Engl, 54:12029-12033.

30. Jiang C, Mei M, Li B et al (2017). A non-viral CRISPR/Cas9 delivery system for therapeutic gene targeting in vivo. Cell Res, 27:440-443.

31. Yang F, Babak T, Shendure J, Disteche CM (2010). Global survey of escape from $\mathrm{X}$ inactivation by RNA-sequencing in mouse. Genome Res, 20:614 622.

32. Gardiner K (1997). Clonability and gene distribution on human chromosome 21: reflections of junk DNA content? Gene, 205:39-46.

33. Song R, Ro S, Michaels JD et al (2009). Many Xlinked microRNAs escape meiotic sex 
chromosome inactivation. Nat Genet, 41:488493.

34. Al Nadaf S, Deakin JE, Gilbert C et al (2012). A cross-species comparison of escape from $\mathrm{X}$ inactivation in Eutheria: implications for evolution of $\mathrm{X}$ chromosome inactivation. Chromosoma, 121:71-78.

35. Heard E (2005). Delving into the diversity of facultative heterochromatin: the epigenetics of the inactive X chromosome. Curr Opin Genet Dev, 15:482-489.

36. Reinius B, Shi C, Hengshuo L et al (2010). Femalebiased expression of long non-coding RNAs in domains that escape $\mathrm{X}$-inactivation in mouse. BMC Genomics, 11:614.

37. Berletch JB, Ma W, Yang F et al (2015). Escape from $\mathrm{X}$ inactivation varies in mouse tissues. PLoS Genet, 11:e1005079.

38. Keown CL, Berletch JB, Castanon R et al (2017). Allele-specific non-CG DNA methylation marks domains of active chromatin in female mouse brain. Proc Natl Acad Sci U $S A$, 114:E2882-E2890.

39. Yu S, Yi H, Wang Z, Dong J (2015). Screening key genes associated with congenital heart defects in Down syndrome based on differential expression network. Int I Clin Exp Pathol, 8:8385-93.

40. Yu W, Liang R, Yang L et al (2007). Screening and identification of human chromosome 21 genes resultingin abnormal development of fetal cerebral cortex with Down syndrome]. Zhonghua YiXue Za Zbi, 87:2759-2763.

41. Sommer CA, Pavarino-Bertelli EC, GoloniBertollo EM et al (2008). Identification of dysregulated genes in lymphocytes from children with Down syndrome. Genome, 51:1929.

42. Gordon J, Amini S, White MK (2013). General overview of neuronal cell culture. Methods Mol Biol, 1078:1-8.

43. Ray B, Chopra N, Long JM, Lahiri DK (2014). Human primary mixed brain cultures: preparation, differentiation, characterization and application to neuroscience research. Mol Brain, 7:63.

44. Braudeau J, Dauphinot L, Duchon A et al (2011). Chronic Treatment with a Promnesiant GABAA $\alpha 5$-Selective Inverse Agonist Increases Immediate Early Genes Expression during
Memory Processing in Mice and Rectifies Their Expression Levels in a DownSyndrome Mouse Model. Adv Pharmacol Sci, 2011:153218.

45. Miyamoto K, Suzuki N, Sakai K et al (2014). A novel mouse model for Down syndrome that harbor a single copy of human artificial chromosome (HAC) carrying a limited number of genes from human chromosome 21. Transgenic Res, 23:317-329.

46. Reddy LH, Sharma R, Chuttani K et al (2005). Influence of administration route on tumor uptake and biodistribution of etoposide loaded solid lipid nanoparticles in Dalton's lymphoma tumor bearing mice. J Control Release, 105:185198.

47. Mout R, Ray M, Yesilbag Tonga G et al (2017). Direct Cytosolic Delivery of CRISPR/Cas9Ribonucleoprotein for Efficient Gene Editing. ACS Nano, 11:2452-2458.

48. Slaymaker IM, Gao L, Zetsche B et al (2016). Rationally engineered Cas9 nucleases with improved specificity. Science, 351:84-88.

49. Kleinstiver BP, Pattanayak V, Prew MS et al (2016). High-fidelity CRISPR-Cas9 nucleases with no detectable genome-wide off-target effects. Nature, 529:490-495.

50. Dow LE, Fisher J, O'rourke KP et al (2015). Inducible in vivo genome editing with CRISPRCas9. Nat Biotechnol, 33:390-394.

51. Solaro R, Chiellini F, Battisti A (2010). Targeted delivery of protein drugs by nanocarriers. Materials (Basel), 3:1928-1980.

52. Bala I, Hariharan S, Kumar MR (2004). PLGA nanoparticles in drug delivery: the state of the art. Crit Rev Ther Drug Carrier Syst, 21:387-422.

53. Weiss CK, Kohnle MV, Landfester $\mathrm{K}$ et al (2008). The First Step into the Brain: Uptake of NIOPBCA Nanoparticles by Endothelial Cells in vitro and in vivo, and Direct Evidence for their Blood-Brain Barrier Permeation. Chem Med Chem, 3:1395-1403.

54. Bose RJ, Lee S-H, Park H (2016). Lipid-based surface engineering of PLGA nanoparticles for drug and gene delivery applications. Biomater Res, 20:34.

55. Wang L, Li Z, Song X et al (2016). Bioinformatic analysis of genes and MicroRNAs associated with atrioventricular septal defect in down syndrome patients. Int Heart J, 57:490-495. 